\title{
Cochrane
}

Cochrane Database of Systematic Reviews

\section{Comparative efficacy and acceptability of pharmacological treatments for insomnia in adults: a systematic review and network meta-analysis (Protocol)}

De Crescenzo F, Foti F, Ciabattini M, Del Giovane C, Watanabe N, Sañé Schepisi M, Quested DJ, Cipriani A, Barbui C, Amato L

De Crescenzo F, Foti F, Ciabattini M, Del Giovane C, Watanabe N, Sañé Schepisi M, Quested DJ, Cipriani A, Barbui C, Amato L.

Comparative efficacy and acceptability of pharmacological treatments for insomnia in adults: a systematic review and network meta-analysis.

Cochrane Database of Systematic Reviews 2016, Issue 9. Art. No.: CD012364.

DOI: 10.1002/14651858.CD012364.

www.cochranelibrary.com

Comparative efficacy and acceptability of pharmacological treatments for insomnia in adults: a systematic review and network metaanalysis (Protocol)

Copyright @ 2016 The Cochrane Collaboration. Published by John Wiley \& Sons, Ltd. 
TABLE OF CONTENTS

HEADER . . . . . . . . . . . . . . . . . . . . . . . . . . . . . . . . . . . . . . . 1

ABSTRACT . . . . . . . . . . . . . . . . . . . . . . . . . . . . . . . . . . . . . . . . .

BACKGROUND . . . . . . . . . . . . . . . . . . . . . . . . . . . . . . . . . . . .

OBJECTIVES . . . . . . . . . . . . . . . . . . . . . . . . . . . . . . . . . . . . . . . . . .

METHODS . . . . . . . . . . . . . . . . . . . . . . . . . . . . . . . . . . . . . .

Figure 1. . . . . . . . . . . . . . . . . . . . . . . . . . . . . . . . . . . . . . . 5

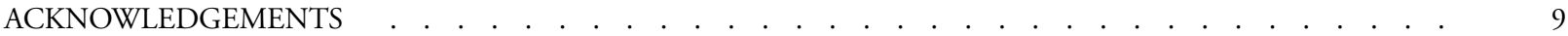

REFERENCES . . . . . . . . . . . . . . . . . . . . . . . . . . . . . . . . . . . . . 10

APPENDICES . . . . . . . . . . . . . . . . . . . . . . . . . . . . . . . . . . . . . . . . . 14

WHAT'S NEW . . . . . . . . . . . . . . . . . . . . . . . . . . . . . . . . . . . . . . 18

CONTRIBUTIONS OF AUTHORS . . . . . . . . . . . . . . . . . . . . . . . . . . . . . . . . . . . . . . . .

DECLARATIONS OF INTEREST . . . . . . . . . . . . . . . . . . . . . . . . . . . . . . . . . . . . . 18

Comparative efficacy and acceptability of pharmacological treatments for insomnia in adults: a systematic review and network metaanalysis (Protocol)

Copyright $\odot 2016$ The Cochrane Collaboration. Published by John Wiley \& Sons, Ltd. 


\title{
[Intervention Protocol]
}

\section{Comparative efficacy and acceptability of pharmacological treatments for insomnia in adults: a systematic review and network meta-analysis}

\author{
Franco De Crescenzo ${ }^{1}$, Francesca Foti ${ }^{2,3}$, Marco Ciabattini ${ }^{4}$, Cinzia Del Giovane ${ }^{5}$, Norio Watanabe ${ }^{6}$, Monica Sañé Schepisi ${ }^{7}$, Digby \\ J Quested $^{8}$, Andrea Cipriani ${ }^{9}$, Corrado Barbui ${ }^{10}$, Laura Amato ${ }^{11}$ \\ ${ }^{1}$ Institute of Psychiatry and Psychology, Catholic University of the Sacred Heart, Rome, Italy. ${ }^{2}$ Department of Psychology, "Sapienza” \\ University of Rome, Rome, Italy. ${ }^{3}$ IRCCS Santa Lucia Foundation, Rome, Italy. ${ }^{4}$ Public Health, Tor Vergata University, Rome, Italy. \\ ${ }^{5}$ Italian Cochrane Centre, Department of Diagnostic, Clinical and Public Health Medicine, University of Modena and Reggio Emilia, \\ Modena, Italy. ${ }^{6}$ Department of Health Promotion and Human Behavior, Kyoto University Graduate School of Medicine / School of \\ Public Health, Kyoto, Japan. ${ }^{7}$ Clinical Epidemiology, National Institute for Infectious Diseases, IRCCS L. Spallanzani, Rome, 00149, \\ Italy. ${ }^{8}$ Department of Psychiatry and Mental Health, Oxford Health NHS Trust, Oxford, UK. ${ }^{9}$ Department of Psychiatry, University \\ of Oxford, Oxford, UK. ${ }^{10}$ Neuroscience, Biomedicine and Movement Sciences, Section of Psychiatry, University of Verona, Verona, \\ Italy. ${ }^{11}$ Department of Epidemiology, Lazio Regional Health Service, Rome, Italy
}

Contact address: Franco De Crescenzo, Institute of Psychiatry and Psychology, Catholic University of the Sacred Heart, L.go A. Gemelli 8, Rome, 00168, Italy. decrescenzo.franco@gmail.com.

Editorial group: Cochrane Common Mental Disorders Group.

Publication status and date: Edited (no change to conclusions), published in Issue 9, 2016.

Citation: De Crescenzo F, Foti F, Ciabattini M, Del Giovane C, Watanabe N, Sañé Schepisi M, Quested DJ, Cipriani A, Barbui C, Amato L. Comparative efficacy and acceptability of pharmacological treatments for insomnia in adults: a systematic review and network meta-analysis. Cochrane Database of Systematic Reviews 2016, Issue 9. Art. No.: CD012364. DOI: 10.1002/14651858.CD012364.

Copyright (C) 2016 The Cochrane Collaboration. Published by John Wiley \& Sons, Ltd.

\section{A B S T R A C T}

This is the protocol for a review and there is no abstract. The objectives are as follows:

1) To compare individual pharmacological treatments for insomnia in adults in terms of:

- efficacy, measured as self-rated quality of sleep or satisfaction with sleep; and

- acceptability of treatment.

2) To generate a clinically-useful hierarchy of available pharmacological treatments for insomnia in adults, according to their efficacy and acceptability.

\section{Description of the condition}

Insomnia is characterised by difficulty in initiating and maintain-

B A C K G R O N D ing sleep, by early morning awakenings and by non-restorative

Comparative efficacy and acceptability of pharmacological treatments for insomnia in adults: a systematic review and network metaanalysis (Protocol)

Copyright $\odot 2016$ The Cochrane Collaboration. Published by John Wiley \& Sons, Ltd. 
sleep, which leads to a condition of daytime blunting. Sleep disorders can lead to a reduction in cognitive, social-emotional, or occupational functioning.

Typical symptoms of insomnia related to sleep (i.e. difficulty initiating sleep, difficulty maintaining sleep, early morning awakenings, non-restorative sleep) are widespread, with an average point prevalence affecting about a third of the population. The prevalence tends to fall to $10 \%$ to $15 \%$ of the population when considering daytime impairment, and the prevalence falls further to a range of $6 \%$ to $10 \%$ if the main sets of diagnostic criteria are considered (Ohayon 2002; Morin 2006; Ohayon 2009). Daytime impairment is expressed in terms of the impact on daytime activities, in cognitive, social, and emotional domains (Léger 2010a). Recent studies have shown that individuals with insomnia exhibit impaired performance in several cognitive functions, including working and episodic memory as well as some aspects of executive functioning (Fortier-Brochu 2012; Bonnet 2014; Shekleton 2014). Cognitive impairments have also been associated with hippocampal atrophy (Joo 2014). Daytime impairments result in reduced productivity at work (Bolge 2009), reduced quality of life (Léger 2012), an increased rate of accidents at work (Kessler 2012) and increased economic burden (Léger 2010b; Rosekind 2010). Indeed, insomnia has major public health implications, representing a significant burden to society. The cumulative expenditures for insomnia include direct costs - such as outpatient encounters and medical procedures, prescription and treatment costs, transportation to and from treatments - and indirect costs - such as healthcare utilisation, lost workplace productivity and increased risk of accidents (Wickwire 2015). Several diseases are correlated with insomnia, particularly hypertension and cardiovascular disorders (Schwartz 1999; Vgontzas 2009a), type 2 diabetes (Vgontzas $2009 \mathrm{~b}$ ) and psychiatric disorders, such as depression and anxiety disorders (Riemann 2007; Baglioni 2011). Drug and alcohol abuse are also associated with insomnia (Johnson 2001; Shibley 2008). To diagnose insomnia, the most commonly-used instruments are the Diagnostic and Statistical Manual of Mental Disorders (DSM IV-TR, DSM-5), the International Classification of Diseases (ICD-10) and the International Classification of Sleep Disorders (ICDS-2, ICSD-3). Together with disrupted sleep and daytime impairment, the DSM-IV-TR sets a minimum duration of one month to diagnose the condition, and the ICD-10 requires symptom occurrence of at least 3 times a week for a month. In addition, the ICD-10 requires the presence of marked personal distress or interference with personal functioning. Both the ICDS3 and the DSM-5 establish a duration of at least three months, with a disturbance frequency of at least 3 times per week despite adequate conditions for sleep, and the absence of co-existing other sleep disorders, mental disorders or medical conditions (WHO 1992; APA 2000; AASM 2005; APA 2013; AASM 2014).

Studies which have compared the main diagnostic systems have shown that the diagnosis of insomnia obtained through their use fails to adequately represent the actual extent of the disease in the population (Ohayon 2009). In particular, it has been observed that, in the same population, the diagnoses obtained by the ICD10 are very low in number compared to those obtained by the DSM-IV-TR or the ICDS-2. Roth 2011 suggested that the DSMIV-TR was superior to other diagnostic systems. A recent study showed that the prevalence of insomnia diagnoses is estimated to have been reduced by half with the transition from DSM-IV-TR to DSM-5 (Chung 2015). Some researchers identified the measure 'global sleep dissatisfaction' - which considers duration, quality of sleep, or both - as an important element which should be included among diagnostic criteria to improve the accuracy and reliability of the diagnosis (Ohayon 2009; Ohayon 2012).

\section{Description of the intervention}

Insomnia treatment is based on sleep hygiene, cognitive behavioural therapy for insomnia (CBT-I) and pharmacological therapy (NICE 2014).

Sleep hygiene refers to a list of behavioural rules designed to increase the likelihood of sleeping well. Poor sleep hygiene can contribute to insomnia, but not cause it; for this reason sleep hygiene education is a necessary, but not a sufficient, treatment for insomnia (Stepanski 2003).

CBT-I is a weekly psychological intervention, normally lasting 8 to 10 weeks. It consists of sleep hygiene instructions, stimulus control therapy, sleep restriction therapy, relaxation and cognitive therapy (Perlis 2005). CBT-I has been shown to be effective in acute treatment as well as in long-term follow-up (Riemann 2009). The combination of CBT-I and pharmacotherapy has been proved to be more effective than CBT-I alone (Morin 2012). CBT-I is often not easily accessible and the prescription of sleep medications is therefore increasing (Ford 2014).

Pharmacotherapy for insomnia consists of different types of drugs. The most commonly-used drugs at present are benzodiazepine receptor agonists (BZRAs) (Ilyas 2012; Ford 2014), which are subdivided into benzodiazepines and benzodiazepine-like drugs (also known as 'Z-drugs'). Other drugs for treating insomnia include antidepressants (mostly tricyclic antidepressants (TCAs)), melatoninergic drugs and orexin receptor antagonists.

BZRAs are positive allosteric modulators at the GABA-A receptor. Gamma-aminobutyric acid (GABA) is the principal inhibitory neurotransmitter of the central nervous system and it is the physiological ligand for GABA-A receptors, which are ligand-gated ion channels. BZRAs' action on the GABA-A receptor is self-limiting, depending on the presence of GABA. In fact, in the absence of GABA, BZRAs cannot open the chloride channel (Rudolph 2011). This self-limiting action is a main reason for the higher safety profile of BZRAs in comparison to previously-used drugs for insomnia, such as barbiturates (Fischbach 1983). Benzodiazepines bind non-selectively onto GABA-A receptors $\alpha 1, \alpha 2, \alpha 3$, or $\alpha 5$ subunits. Benzodiazepine-like drugs generally have a short halflife, which grants few daytime adverse effects (Rudolph 2011). 
TCAs act as inhibitors of serotonin and norepinephrine reuptake and they have also anticholinergic and antihistaminergic properties; it is supposed that the antihistaminergic action is the main reason for their sedating properties (Richelson 1979; Ware 1983). Melatoninergic drugs are divided into exogenous melatonin and melatonin receptor agonists. Melatonin is an endogenous hormone secreted by the pineal gland and involved in circadian rhythms and the sleep/wake cycle. Exogenous melatonin and melatonin receptor agonists bind MT1 and MT2 melatonin receptors, which regulate the sleep-wake cycle and inhibit arousal signals (Dubocovich 2005; Kato 2005).

Orexin receptor antagonists are a new class of drugs under development. The orexins are excitatory neuropeptides, which convey their actions on two G protein-coupled receptors called OX1 and OX2. They are involved in the sleep/wake cycle, and it has been demonstrated that individuals who suffer from narcolepsy show a deficit of neurons producing orexins (Sakurai 2005).

\section{How the intervention might work}

BZRAs are a common choice for insomnia treatment and their use has been systematically increasing over the years (Ford 2014). Benzodiazepines are efficacious in terms of sleep onset latency (SOL) and total sleep time (TST), and they are used clinically for different types of insomnia: short-acting medications are indicated for patients with SOL, while longer-acting medications are preferable for patients with sleep maintenance insomnia (Holbrook 2000; Buscemi 2007). Even though BZRAs have been shown to be effective in the acute treatment of insomnia, they cause important side effects such as cognitive and motor impairments, and somnolence (Holbrook 2000; Poyares 2004; Buscemi 2007; Wafford 2008). In particular, long duration therapies with benzodiazepines may result in the appearance of dependency, withdrawal symptoms (e.g. rebound insomnia) and worsening of sleep parameters (Allison 2003; Poyares 2004). Benzodiazepine-like drugs (i.e. eszopiclone; zaleplon; zolpidem; zopiclone) have a short half-life, produce fewer next-day cognitive and motor impairments (Wafford 2008; Nutt 2010), and at present their clinical use is preferred to benzodiazepines (Wafford 2008; Ford 2014).

Antidepressants are widely used for the treatment of insomnia, and their prescription appears to increase over time together with other non-benzodiazepine drugs (Ford 2014). However, among antidepressants, only doxepin has been approved for the treatment of insomnia and the prescription of other antidepressants (e.g. trazodone, mirtazapine, amytriptiline) is off-label. Doxepin inhibits serotonin and norepinephrine reuptake and inactivates cholinergic, histaminergic and alpha1-adrenergic receptors. At low dose (less than 10mg/day), doxepin has little effect on the serotonergic and adrenergic receptors, promoting sleep onset and duration, and acting as a selective histamine $\mathrm{H} 1$ receptor antagonist (Yeung 2015). Therapeutic effects of doxepin are observed at very low dosages ( $3 \mathrm{mg}$ to $6 \mathrm{mg} /$ day), improving sleep maintenance without rebound insomnia or physical dependence (Hajak 2001). Common side effects include sedation, nasopharyngitis, gastrointestinal effects, and hypertension (Weber 2010). Doxepin has also been demonstrated to be effective for sleep maintenance and early morning awakenings, which are the most common insomnia-related complaints in the elderly (Krystal 2010).

Melatonin receptor agonists, such as melatonin and ramelteon, have been demonstrated to be a well-tolerated option for the treatment of patients with insomnia characterised by difficulty in sleep onset (Simpson 2008). Ramelteon was associated with reduced subjective sleep latency and improved sleep quality, but not with increased subjective total sleep time. Ramelteon was also associated with improvement in sleep efficiency, and total sleep time by polysomnography, without significant side effects other than somnolence (Kuriyama 2014). At present, no study has demonstrated clear effectiveness for melatoninergic drugs in insomnia.

There are many orexin receptor antagonists under investigation for the treatment of insomnia, and they can be divided into single orexin receptor antagonists (SORAs) and dual orexin receptor antagonists (DORAs) (Equihua 2013). Thus far, the Food and Drug Administration (FDA) has approved only suvorexant, which belongs to the DORAs category, for the treatment of insomnia. In a recent double-blind, placebo-controlled trial, patients undergoing suvorexant therapy showed improved subjective TST and subjective SOL compared with placebo. Those improvements were noticeable after one week of treatment and were maintained throughout one year. The drug was well tolerated by insomnia patients and the most commonly-reported adverse effects were daytime somnolence and fatigue (Michelson 2014).

Other drugs approved for the treatment of insomnia are barbiturates - chloral hydrate, ethchlorvynol, triclofos sodium - but they are not used clinically any longer due to their important adverse effects, toxic effects, and risk of misuse and dependence (Morin 2012; Mowry 2013).

Off-label drugs include antidepressants (with the exception of doxepin which has an indication for the treatment of insomnia) and antipsychotics, which are used for the treatment of insomnia due to psychiatric comorbidities and are considered as a second line treatment (Morin 2012; Saddichha 2010). Antihistamines are still found in many over-the-counter (OTC) sleep aids (Risberg 1975). Most OTCs are non-selective, having anti-muscarinic, anti-adrenergic properties and acting on dopamine and serotonin receptors, which gives rise to unacceptable side effects. Indeed, antihistamines used as sleep-inducing agents can cause drowsiness, but the evidence for their efficacy is very limited and the data on safety and tolerance now discourage their use in insomnia (Morin 2005; Morin 2012; NICE 2014).

\section{Why it is important to do this review}

Previous pairwise meta-analyses could not generate clear hierarchies for the efficacy and acceptability of available treatments. 
Some Cochrane intervention reviews of sleep drugs for insomnia are in progress (Everitt 2013; Rösner 2013a; Rösner 2013b; Rösner 2013c; Rösner 2013d; Moriichi 2014; Takeshima 2014), while some non-Cochrane systematic reviews have been undertaken (Holbrook 2000; Dündar 2004; Buscemi 2007; Winkler 2014), but this will be the first network meta-analysis in the field. Our intention is to reduce the uncertainty about the efficacy of treatments due to the limited number of direct comparisons as reported in previous standard meta-analyses, and provide an evidence-based hierarchy of the comparative efficacy and acceptability of the drugs approved for the treatment of primary insomnia. This network meta-analysis will help clinicians, patients and policy makers to make informed decisions on the best pharmacological treatments for insomnia. In conclusion, the present review will synthesise the best available clinical evidence, including both direct and indirect comparisons, in order to help clinicians and patients to make informed decisions on the best pharmacological treatments approved for insomnia in terms of efficacy and acceptability.

\section{O B J E C T I VES}

1) To compare individual pharmacological treatments for insomnia in adults in terms of:

- efficacy, measured as self-rated quality of sleep or satisfaction with sleep; and

- acceptability of treatment.

2) To generate a clinically-useful hierarchy of available pharmacological treatments for insomnia in adults, according to their efficacy and acceptability.

\section{METHODS}

\section{Criteria for considering studies for this review}

\section{Types of studies}

We will include randomised controlled trials (RCTs) comparing active drugs with other active drugs and/or placebo as oral therapy in the treatment of primary insomnia. We will exclude controlled clinical trials, cluster-randomised trials and cross-over trials, in order to avoid possible sources of heterogeneity.

\section{Types of participants}

\section{Participant characteristics}

Adults aged 18 or older will be included. There will be no limits in terms of gender or ethnicity.

\section{Diagnosis}

Insomnia diagnosed according to any standardised diagnostic criteria, such as the DSM-III (APA 1980), DSM-III-R (APA 1987), DSM-IV (APA 1994), DSM-IV TR (APA 2000), DSM-5 (APA 2013), ICD-10 (WHO 1992), International Classification of Sleep Disorders (ICSD) (AASD 1990), ICSD-2 (AASM 2005) or ICSD-3 (AASM 2014).

\section{Co-morbidities}

We will include studies on primary insomnia and exclude those considering patients with insomnia due to psychiatric or physical comorbidity. The distinction between primary and secondary insomnia is important for a network meta-analysis, because the severity and the pathophysiologic heterogeneity of the disturbances that cause insomnia are likely to be strong confounders interfering with the reliability of the results. Moreover, the diagnosis has important implications for treatment: therapy for primary insomnia focuses on the improvement of sleep, while therapy for secondary insomnia focuses on the causative medical problem, which also implies that the dose and types of drugs may not be comparable.

\section{Setting}

We will consider studies performed in any setting.

\section{Types of interventions}

We will include RCTs that evaluate one or more of the following pharmacological interventions as monotherapy, compared to placebo and/or to another active agent:

- Antidepressants: amitriptyline; doxepin; mirtazapine; trazodone;

- Benzodiazepines: brotizolam; clonazepam; diazepam; estazolam; flunitrazepam; flurazepam; haloxazolam; loprazolam; lorazepam; lormetazepam; midazolam; nimetazepam; nitrazepam; quazepam; rilmazafone; temazepam; triazolam;

- Benzodiazepine-like agents: eszopiclone; zaleplon; zolpidem; zopiclone;

- Melatoninergic drugs: melatonin; ramelteon;

- Orexin receptor antagonists: suvorexant.

We will exclude barbiturates, chloral hydrate, ethchlorvynol, triclofos sodium and quetiapine due to their important adverse effects, toxic effects, and risk of misuse and dependence (Morin 
2012; Mowry 2013). We will also exclude herbal products and medical devices.

Figure 1 shows the network of all possible pairwise comparisons between the eligible treatments. We assume that any patient who meets the inclusion criteria is, in principle, equally likely to be randomised to any of the eligible treatments.

Figure I. Figure I represents all possible pairwise comparisons from the network of the interventions.



\section{Comparability of dosages}

We will include only studies randomising patients to drugs within the therapeutic dose. Both fixed-dose and flexibledose designs will be allowed. We will establish therapeutic doses according to the British National Formulary (BNF) (www.medicinescomplete.com). There is the possibility that some trials may compare one agent at the upper limit of its therapeutic range with another agent at the lower limit of its therapeutic range within the same study. We may look at heterogeneity and then add a binary variable (yes/no) to report if dosages are comparable and use this information for analysis.

We will exclude: (i) combination treatments; (ii) augmentation studies (e.g. drug A+ drug B versus drug A); (iii) all non-pharmacological treatments.

Comparative efficacy and acceptability of pharmacological treatments for insomnia in adults: a systematic review and network meta- 


\section{Types of outcome measures}

Studies that meet the above inclusion criteria will be included regardless of whether they report on the following outcomes.

\section{Primary outcomes}

1. Quality of sleep or satisfaction with sleep index (as a continuous outcome), as measured by any self-rated validated scale, such as the Pittsburgh Sleep Quality Index (PSQI) (Buysse 1989), the Insomnia Severity Index (ISI) (Bastien 2001), or the Leeds Sleep Evaluation Questionnaire (LSEQ) (Parrott 1978; Zisapel 2003). In case other standardised scales were used by some trials, we will use them in the absence of PSQI, ISI or LSEQ.

2. Drop-outs for any reason.

\section{Secondary outcomes}

1. Drop-outs due to any adverse event.

2. Daytime functioning as measured by attentional tasks, tests or any self-rated measure of function, for example the 36-item short-form (SF-36) (Ware 1992), the Stanford Sleepiness Scale (SSS) (Hoddes 1973) or the Epworth Sleepiness Scale (ESS) (Johns 1991).

3. Sleep onset latency evaluated by polysomnography, defined as the length of time (in minutes) after lights-out until sleep onset.

4. Wake time after sleep onset (WASO) evaluated by polysomnography, defined as the length of time (in minutes) of wakefulness after the onset of persistent sleep.

5. Total sleep time (TST) evaluated by polysomnography, defined as the total time (in minutes) a person spent sleeping during the in-bed interval. TST is time in bed minus SOL and minus WASO.

\section{Timing of outcome assessment}

We will consider outcomes assessed at four weeks post-treatment or at its closest time point. We will include trials with an assessment from one week up to three months. Separately, we will also consider long-term outcomes (more than 3 months).

\section{Hierarchy of outcome measures}

For the primary outcome "Quality of sleep" we will select first the PSQI scale firstly, second the ISI scale and third the LSEQ scale. Measures of daytime functioning will be considered and analysed separately.

\section{Search methods for identification of studies}

\section{Electronic searches}

\section{Bibliographic databases}

We will search the following bibliographic databases for reports of RCTs using relevant subject headings (controlled vocabularies) and search syntax, appropriate to each resource (Appendix 1):

- Cochrane Central Register of Controlled Trials

(CENTRAL, all years)

- Ovid MEDLINE (1950 onwards)

- PubMed (current year)

- Ovid Embase (1980 onwards)

- Ovid PsycINFO (all years)

We will not restrict our search by language, date or publication status.

We will conduct a separate search to identify other systematic reviews and meta-analyses (on Ovid MEDLINE; the Cochrane Database of Systematic Reviews (CDSR); the Database of Abstracts of Reviews of Effects (DARE); and Epistemonikos)

\section{International trial registries}

We will search the World Health Organization's trials portal ( ICTRP) and ClinicalTrials.gov to identify unpublished or ongoing studies.

\section{Searching other resources}

\section{Reference lists}

We will screen the reference lists of all included studies and relevant systematic reviews to identify additional studies missed from the original electronic searches (for example unpublished or in-press citations).

\section{Correspondence}

We will contact trialists and subject experts for information on unpublished or ongoing studies or to request additional trial data.

\section{Data collection and analysis}

\section{Selection of studies}

Two review authors (FDC, MC) will independently screen titles and abstracts retrieved by the search strategy. Full-texts of potentially relevant studies will then be assessed independently by two 
authors (FDC, MC). Disagreements will be resolved through discussion with a third member of the review team (LA).

\section{Data extraction and management}

We will use a data collection form to extract study characteristics and outcome data, which has been piloted on at least one study in the review. Two review authors (FDC, MC) will independently extract study characteristics and outcome data from included studies, as follows:

Methods: first author or acronym, year of publication, publication (full-text publication, abstract publication, unpublished data), study design.

Participants: diagnosis, sample size $(\mathrm{N})$, mean age, gender distribution, severity of illness, treatment setting.

Interventions: number of patients allocated to each arm, drug name, dose, route or administration, duration of the interventions and follow-up.

Outcomes: primary and secondary outcomes evaluated.

Adverse events (AEs): AEs as unfavourable symptoms occurring during the course of the study.

Notes: country, funding source; investigational drug versus comparator.

We will note in the 'Characteristics of included studies' table if outcome data were not reported in a usable way. We will resolve disagreements by consensus or by involving a third person (LA). Two review authors (FDC, MC) will enter data into Review Manager (RevMan 2014). We will double-check that data are entered correctly by comparing the data presented in the systematic review with the study reports.

Data on potential effect modifiers

We will extract from each included study data that may act as effect modifiers: age, funding source, studies reported as high risk of bias.

Outcome data

We will extract from each included study:

- self-rated quality of sleep scale, as a continuous outcome: mean and standard deviation (SD);

- drop-outs for any reason: number of participants who dropped out for any reason, of the total number of participants randomised to each arm;

- drop-outs due to any adverse events: number of participants who dropped out because of any adverse event, of the total number of participants randomised to each arm;

- daytime functioning; each single scale will be analysed separately as a continuous outcome: mean and SD;

- polysomnographic outcomes SOL, WASO and TST: mean in minutes and SD.

\section{Assessment of risk of bias in included studies}

Two review authors (FDC, MC) will independently assess the risk of bias of each study, using the criteria outlined in the Cochrane
Handbook for Systematic Reviews of Interventions (Higgins 2011). We will resolve any disagreements by discussion or by involving another author (LA). The following domains will be assessed: random sequence generation, allocation concealment, blinding of providers and participants, blinding of outcome assessment, incomplete outcome data, and selective outcome reporting. We will judge each potential source of bias as high, low or unclear and provide a supporting quotation from the study report together with a justification for our judgment in the 'Risk of bias' table. We will report the 'Risk of bias' judgements across different studies for each of the domains listed. Where information on risk of bias relates to unpublished data or correspondence with a trialist, we will note this in the 'Risk of bias' table. A judgement of high risk of bias in one or more domain will be considered as a 'high risk' study, a judgement of low risk of bias in all domains will be considered as a 'low risk' study, and a judgement of unclear risk of bias in one or more domains as an 'unclear risk' study. When considering treatment effects, we will take into account the risk of bias for the studies that contribute to that outcome.

\section{Measures of treatment effect}

\section{Relative treatment effects}

\section{Dichotomous data}

Dichotomous outcomes will be analysed by calculating the relative risk (RR) for each trial with the uncertainty in each result being expressed by its $95 \%$ confidence interval (CI).

\section{Continuous data}

Continuous outcomes will be analysed by calculating the mean difference (MD) with the relative 95\% CI when the study used the same instruments for assessing the outcome. We will use the standardised mean difference (SMD) when studies used different instruments.

\section{Relative treatment ranking}

For any primary outcome we will also estimate the ranking probabilities for all treatments of being at each possible rank for each intervention. Then we will obtain a treatment hierarchy using the surface under the cumulative ranking curve (SUCRA) and mean ranks. SUCRA will be expressed as a percentage and is interpreted as the percentage of efficacy or safety a treatment achieves in relation to a treatment that would be ranked first without uncertainty (Salanti 2011). 


\section{Unit of analysis issues}

For simple pairwise meta-analysis, if all arms in a multi-arm trial are to be included in the meta-analysis and one treatment arm is to be included in more than one treatment comparison, then we will divide the number of events and the number of participants in that arm by the number of treatment comparisons made. This method will avoid the multiple use of participants in the pooled estimate of treatment effect, while retaining information from each arm of the trial. It will, however, compromise the precision of the pooled estimate slightly. In the network meta-analysis, we account for the correlation between the effect sizes from multi-arm studies (Higgins 2011, chapter 16.5.4).

\section{Dealing with missing data}

We will contact study authors when there are missing or unclear data. If dichotomous outcome data are still missing, they will be managed according to the intention-to-treat (ITT) principle, and we will assume that patients who dropped out after randomisation had a negative outcome. Missing continuous outcome data will either be analysed using the last observation carried forward to the final assessment (LOCF) or, if LOCF data are reported by the trial authors, will be analysed on an endpoint basis, including only participants with a final assessment. When P values, t-values, CIs or standard errors are reported in articles, we will calculate SDs from their values (Furukawa 2006).

\section{Assessment of heterogeneity}

In the context of the network meta-analysis, we will assume a common within-network heterogeneity and the generalised Qstatistic estimator will be used for the heterogeneity variance.

\section{Assessment of clinical and methodological heterogeneity within treatment comparisons}

To evaluate the presence of heterogeneity deriving from different trial designs or different clinical characteristics of study participants, we will generate descriptive statistics for trial and study population characteristics across all eligible trials that compare each pair of interventions. We will assess the presence of clinical heterogeneity within each pairwise comparison by comparing these characteristics.

\section{Assessment of transitivity across treatment comparisons}

We expect that the transitivity assumption will hold assuming that all pairwise comparisons do not differ on average with respect to the distribution of effect modifiers (e.g. age). The assumption of transitivity will be evaluated in each primary outcome by comparing the clinical and methodological characteristics (potential effect modifiers presented in Data extraction and management) across the different pairwise comparisons.

\section{Assessment of reporting biases}

The possibility of reporting bias will be evaluated for each primary outcome by means of the contour-enhanced funnel plots if enough studies (at least 10) are available (Peters 2008). These are funnel plots showing areas of statistical significance and they can help to distinguish publication bias from other possible reasons for asymmetry. In a network of interventions each study estimates the relative effect of different interventions, so asymmetry in the funnel plot cannot be judged. To account for this, we will use an adaptation of the funnel plot by subtracting from each study-specific effect size the mean of meta-analysis of the studyspecific comparison and plot it against the study's standard error (Chaimani 2012; Chaimani 2013). We will draw the comparisonadjusted funnel plot for all placebo-controlled trials (if at least 10 trials are available). Any asymmetry in the plot indicates the presence of small study effects and not necessarily reporting bias.

\section{Data synthesis}

\section{Methods for direct treatment comparisons}

We will perform conventional pairwise meta-analyses for primary and secondary outcomes using a random-effects model in RevMan for every treatment comparison with at least two studies (DerSimonian 1986).

\section{Methods for indirect and mixed comparisons}

We will perform network meta-analysis (NMA) for primary outcomes. NMA is a method of synthesising information from a network of trials addressing the same question but involving different interventions (Cipriani 2013). NMA combines direct evidence (from studies comparing two treatments, e.g. A versus B) and indirect evidence (e.g. the comparison A versus B comes from studies comparing $\mathrm{A}$ and $\mathrm{B}$ versus a common comparator $\mathrm{C}$ ) across a network of randomised trials into a single effect size, and under certain assumptions it can increase the precision in the estimates while randomisation is respected. We will perform NMA using a random-effects model within a frequentist setting assuming equal heterogeneity across all comparisons, and we will account for correlations induced by multi-arm studies (Lu 2006; Salanti 2009). The models will enable us to estimate the probability of each intervention being the best for each outcome, given the relative effect sizes as estimated in NMA. We will perform NMA in Stata 13 using the 'mvmeta' command and self-programmed Stata routines available at http://www.mtm.uoi.gr (Chaimani 2014; White 2011; White 2012).

Results of meta-analysis and NMA will be applied when reasonable and presented as summary relative effect sizes (MD, SMD or RR) for each possible pair of treatments. 
Subgroup analysis and investigation of heterogeneity

\section{Measures and tests for heterogeneity}

We will statistically assess the presence of heterogeneity for all direct pairwise comparisons using the $\tau^{2}$. The assessment of statistical heterogeneity in the entire network will be based on the magnitude of the heterogeneity variance parameter $\left(\tau^{2}\right)$ estimated from the NMA models. We will compare the magnitude of the heterogeneity variance with the empirical distribution as derived by Turner (Turner 2012). We will also estimate a total $\mathrm{I}^{2}$ value for heterogeneity in the network as described elsewhere (Jackson 2014).

\section{Assessment of statistical inconsistency}

Consistency in a network of treatments refers to the agreement between direct and indirect evidence on the same comparisons. Joint analysis can be misleading if the network is substantially inconsistent. Inconsistency can be present if the trials in the network have very different protocols and their inclusion/exclusion criteria are not comparable or may result as an uneven distribution of the effect modifiers across groups of trials that compare different treatments.

\section{Local approaches for evaluating inconsistency}

We will first check for any erroneous data abstraction. Then, to evaluate the presence of inconsistency locally, we will use the loopspecific approach. This method evaluates the consistency assumption in each closed loop of the network separately as the difference between direct and indirect estimates for a specific comparison in the loop (inconsistency factor) (Veroniki 2013). The magnitude of the inconsistency factors and their 95\% CIs can then be used to infer as to the presence of inconsistency in each loop. We will assume a common heterogeneity estimate within each loop. We will present the results of this approach graphically in a forest plot using the 'ifplot' command in Stata (Chaimani 2013).

\section{Investigation of heterogeneity and inconsistency}

If sufficient studies are available, we will perform network subgroup analyses for the primary efficacy outcome by using age (over
65 years old versus 18 to 65 years old) as possible sources of inconsistency or heterogeneity (Floyd 2000; Ohayon 2002; Ancoli-Israel 2008; Fetveit 2009).

\section{Sensitivity analysis}

If enough studies per comparison are identified, we will carry out a sensitivity analysis of the primary outcomes including only trials at low risk of bias in all domains. Moreover, we will perform a sensitivity analysis to assess the robustness of the results if imputations have been applied.

\section{'Summary of findings' table}

The main results of the review will be presented in 'Summary of findings' (SoF) tables, as recommended by Cochrane (Schünemann 2011). We will produce the SoF tables for estimates from the NMA based on the methodology developed from the GRADE Working Group (GRADE 2004). For more details, see Salanti 2014. We will include an overall grading of the evidence for the following main outcomes:

Efficacy:

- Self-rated quality of sleep or satisfaction with sleep.

Acceptability:

- Drop-outs for any reason.

We will grade quality of the evidence considering study limitations, indirectness, inconsistency, imprecision of effect estimates, and risk of publication bias. According to the software GRADEpro GDT 2014, we will assign four levels of quality of evidence: high, moderate, low, very low.

\section{ACKNOWLEDGEMENTS}

We thank Dr G. Della Marca (Catholic University of the Sacred Heart) for his expert advice.

CRG Funding Acknowledgement:

The National Institute for Health Research (NIHR) is the largest single funder of the Cochrane Common Mental Disorders Group.

\section{Disclaimer:}

The views and opinions expressed herein are those of the authors and do not necessarily reflect those of the NIHR, National Health Service (NHS) or the Department of Health. 


\section{R E F E R E N C E S}

\section{Additional references}

\section{AASD 1990}

American Association of Sleep Disorders. International Classification of Sleep Disorders: Diagnostic and Coding Manual. Rochester (MN): American Association of Sleep Disorders, 1990

\section{AASM 2005}

American Academy of Sleep Medicine. International Classification of Sleep Disorders. Diagnostic and Coding Manual. 2nd Edition. Westchester: American Academy of Sleep Medicine, 2005.

\section{AASM 2014}

American Academy of Sleep Medicine. International Classification of Sleep Disorders. Diagnostic and Coding Manual. 3rd Edition. Chicago: American Academy of Sleep Medicine, 2014.

Allison 2003

Allison C, Pratt JA. Neuroadaptive processes in GABAergic and glutamatergic systems in benzodiazepine dependence. Pharmacology \& Therapeutics 2003;98(2):171-95.

Ancoli-Israel 2008

Ancoli-Israel S, Ayalon L, Salzman C. Sleep in the elderly: normal variations and common sleep disorders. Harvard Review of Psychiatry 2008;16(5):279-86.

\section{APA 1980}

American Psychiatric Association. Diagnostic and Statistical Manual of Mental Disorders (DSM-III). 3rd Edition.

Washington, DC: American Psychiatric Association, 1980.

\section{APA 1987}

American Psychiatric Association. Diagnostic and Statistical Manual of Mental Disorders (DSM-III-R). 3rd Edition. Washington, DC: American Psychiatric Association, 1987.

\section{APA 1994}

American Psychiatric Association. Diagnostic and Statistical Manual of Mental Disorders (DSM-IV). 4th Edition.

Washington, DC: American Psychiatric Association, 1994.

\section{APA 2000}

American Psychiatric Association. Diagnostic and Statistical Manual of Mental Disorders (DSM-IV-TR). 4th Edition. Washington, DC: American Psychiatric Association, 2000.

APA 2013

American Psychiatric Association. Diagnostic and Statistical Manual of Mental Disorders (DSM-V). 5th Edition.

Washington, DC: American Psychiatric Association, 2013.

\section{Baglioni 2011}

Baglioni C, Battagliese G, Feige B, Spiegelhalder K, Nissen C, Voderholzer U, et al. Insomnia as a predictor of depression: a meta-analytic evaluation of longitudinal epidemiological studies. Journal of Affective Disorders 2011; 135(1-3):10-19.

\section{Bastien 2001}

Bastien $\mathrm{CH}$, Vallieres A, Morin CM. Validation of the Insomnia Severity Index as an outcome measure for insomnia research. Sleep Medicine 2001;2(4):297-307.

Bolge 2009

Bolge SC, Doan JF, Kannan H, Baran RW. Association of insomnia with quality of life, work productivity, and activity impairment. Quality of Life Research 2009;18(4):415-22.

\section{Bonnet 2014}

Bonnet MH, Burton GG, Arand DL. Physiological and medical findings in insomnia: implications for diagnosis and care. Sleep Medicine Reviews 2014;18(2):111-22.

Buscemi 2007

Buscemi N, Vandermeer B, Friesen C, Bialy L, Tubman M, Ospina $\mathrm{M}$, et al. The efficacy and safety of drug treatments for chronic insomnia in adults: a meta-analysis of RCTs. Journal of General Internal Medicine 2007;22(9):1335-50.

Buysse 1989

Buysse DJ, Reynolds CF 3rd, Monk TH, Berman SR, Kupfer DJ. The Pittsburgh Sleep Quality Index: a new instrument for psychiatric practice and research. Psychiatry Research 1989;28(2):193-213.

\section{Chaimani 2012}

Chaimani A, Salanti G. Using network meta-analysis to evaluate the existence of small-study effects in a network of interventions. Research Synthesis Methods 2012;3(2): $161-76$.

\section{Chaimani 2013}

Chaimani A, Higgins JP, Mavridis D, Spyridonos P, Salanti G. Graphical tools for network meta-analysis in Stata. PloS One 2013;8(10):e76654.

Chaimani 2014

Chaimani A, Mavridis D, Salanti G. A hands-on practical tutorial on performing meta-analysis with Stata. Evidencebased Mental Health 2014;17:111-6.

\section{Chung 2015}

Chung KF, Yeung WF, Ho FY, Yung KP, Yu YM, Kwok $\mathrm{CW}$. Cross-cultural and comparative epidemiology of insomnia: the Diagnostic and statistical manual (DSM), International classification of diseases (ICD) and International classification of sleep disorders (ICSD). Sleep Medicine 2015;16(4):477-82.

Cipriani 2013

Cipriani A, Higgins JP, Geddes JR, Salanti G. Conceptual and technical challenges in network meta-analysis. Annals of Internal Medicine 2013;159(2):130-7.

DerSimonian 1986 DerSimonian R, Laird N. Meta-analysis in clinical trials. Controlled Clinical Trials 1986;7:177-88.

Dubocovich 2005

Dubocovich ML, Markowska M. Functional MT1 and MT2 melatonin receptors in mammals. Endocrine 2005;27 (2):101-10 


\section{Dündar 2004}

Dündar Y, Dodd S, Strobl J, Boland A, Dickson R, Walley T. Comparative efficacy of newer hypnotic drugs for the short-term management of insomnia: a systematic review and meta-analysis. Human Psychopharmacology 2004;19(5): $305-22$.

Equihua 2013

Equihua AC, De La Herrán-Arita AK, Drucker-Colin R. Orexin receptor antagonists as therapeutic agents for insomnia. Frontiers in Pharmacology 2013;4:163.

\section{Everitt 2013}

Everitt H, Baldwin DS, Mayers A, Malizia AL, Wilson S. Antidepressants for insomnia. Cochrane Database of Systematic Reviews 2013, Issue 10. [DOI: 10.1002/ 14651858.CD010753]

Fetveit 2009

Fetveit A. Late-life insomnia: a review. Geriatrics \& Gerontology International 2009;9(3):220-34.

\section{Fischbach 1983}

Fischbach R. Efficacy and safety of midazolam and vesparax in treatment of sleep disorders. British Journal of Clinical Pharmacology 1983;16(Suppl 1):S167-71.

\section{Floyd 2000}

Floyd JA, Medler SM, Ager JW, Janisse JJ. Age-related changes in initiation and maintenance of sleep: a metaanalysis. Research in Nursing \& Health 2000;23(2):106-17.

\section{Ford 2014}

Ford ES, Wheaton AG, Cunningham TJ, Giles WH, Chapman DP, Croft JB. Trends in outpatient visits for insomnia, sleep apnea, and prescriptions for sleep medications among US adults: findings from the National Ambulatory Medical Care survey 1999-2010. Sleep 2014; 37(8):1283-93.

Fortier-Brochu 2012

Fortier-Brochu E, Beaulieu-Bonneau S, Ivers H, Morin $\mathrm{CM}$. Insomnia and daytime cognitive performance: a metaanalysis. Sleep Medicine Reviews 2012;16(1):83-94.

\section{Furukawa 2006}

Furukawa TA, Barbui C, Cipriani A, Brambilla P, Watanabe $\mathrm{N}$. Imputing missing standard deviations in metaanalyses can provide accurate results.. Journal of Clinical Epidemiology 2006;59:7-10.

\section{GRADE 2004}

Atkins D, Best D, Briss PA, Eccles M, Falck-Ytter Y, Flottorp S, et al. GRADE Working Group. Grading quality of evidence and strength of recommendations. BMJ 2004; 328(7454): 1490 .

GRADEpro GDT 2014 [Computer program] GRADE Working Group, McMaster University. GRADEpro GDT. Hamilton (ON): GRADE Working Group, McMaster University, 2014.

\section{Hajak 2001}

Hajak G, Rodenbeck A, Voderholzer U, Riemann D, Cohrs S, Hohagen F, et al. Doxepin in the treatment of primary insomnia: a placebo-controlled, double-blind, polysomnographic study. Journal of Clinical Psychiatry 2001;62(6):453-63.

\section{Higgins 2011}

Higgins JPT, Green S (editors). Cochrane Handbook for Systematic Reviews of Interventions Version 5.1 [updated March 2011]. The Cochrane Collaboration, 2011.

Available from www.cochrane-handbook.org.

\section{Hoddes 1973}

Hoddes E, Zarcone V, Smythe H, Phillips R, Dement WC. Quantification of sleepiness: a new approach. Psychophysiology 1973;10(4):431-6.

\section{Holbrook 2000}

Holbrook AM, Crowther R, Lotter A, Cheng C, King D. Meta-analysis of benzodiazepine use in the treatment of insomnia. Canadian Medical Association Journal 2000;162 (2):225-33.

\section{Ilyas 2012}

Ilyas S, Moncrieff J. Trends in prescriptions and costs of drugs for mental disorders in England, 1998-2010. British Journal of Psychiatry 2012;200(5):393-8.

\section{Jackson 2014}

Jackson D, Barrett JK, Rice S, White IR, Higgins JP. A design-by-treatment interaction model for network metaanalysis with random inconsistency effects. Statistics in Medicine 2014;33(21):3639-54.

\section{Johns 1991}

Johns MW. A new method for measuring daytime sleepiness: the Epworth sleepiness scale. Sleep 1991;14(6):540-5.

Johnson 2001 Johnson EO, Breslau N. Sleep problems and substance use in adolescence. Drug and Alcohol Dependence 2001;64(1): $1-7$.

Joo 2014

Joo EY, Kim H, Suh S, Hong SB. Hippocampal substructural vulnerability to sleep disturbance and cognitive impairment in patients with chronic primary insomnia: magnetic resonance imaging morphometry. Sleep 2014;37(7):1189-98.

\section{Kato 2005}

Kato K, Hirai K, Nishiyama K, Uchikawa O, Fukatsu K, Ohkawa $S$, et al. Neurochemical properties of ramelteon (TAK-375), a selective MT1/MT2 receptor agonist. Neuropharmacology 2005;48(2):301-10.

Kessler 2012

Kessler RC, Berglund PA, Coulouvrat C, Fitzgerald T, Hajak G, Roth T, et al. Insomnia, comorbidity, and risk of injury among insured Americans: results from the America Insomnia Survey. Sleep 2012;35(6):825-34.

\section{Krystal 2010}

Krystal AD, Durrence HH, Scharf M, Jochelson P, Rogowski R, Ludington E, et al. Efficacy and safety of Doxepin $1 \mathrm{mg}$ and $3 \mathrm{mg}$ in a 12-week sleep laboratory and outpatient trial of elderly subjects with chronic primary insomnia. Sleep 2010;33(11):1553-61. 


\section{Kuriyama 2014}

Kuriyama A, Honda M, Hayashino Y. Ramelteon for the treatment of insomnia in adults: a systematic review and meta-analysis. Sleep Medicine 2014;15(4):385-92.

\section{Lu 2006}

Lu G, Ades A. Assessing evidence inconsistency in mixed treatment comparisons. Journal of American Statistical Association 2006;101:447-59.

\section{Léger 2010a}

Léger D, Partinen M, Hirshkowitz M, Chokroverty S, Touchette E, Hedner J, EQUINOX (Evaluation of daytime QUality Impairment by Nocturnal awakenings in Outpatients eXperience) survey investigator group. Daytime consequences of insomnia symptoms among outpatients in primary care practice: EQUINOX international survey. Sleep Medicine 2010;11(10):999-1009.

\section{Léger 2010b}

Léger D, Bayon V. Societal costs of insomnia. Sleep Medicine Reviews 2010;14(6):379-89.

\section{Léger 2012}

Léger D, Morin CM, Uchiyama M, Hakimi Z, Cure S, Walsh JK. Chronic insomnia, quality-of-life, and utility scores: comparison with good sleepers in a cross-sectional international survey. Sleep Medicine 2012;13(1):43-51.

\section{Michelson 2014}

Michelson D, Snyder E, Paradis E, Chengan-Liu M, Snavely DB, Hutzelmann J, et al. Safety and efficacy of suvorexant during 1-year treatment of insomnia with subsequent abrupt treatment discontinuation: a phase 3 randomised, double-blind, placebo-controlled trial. Lancet Neurology 2014;13(5):461-71.

\section{Moriichi 2014}

Moriichi A, Tomita N, Sado M, Ota E, Mori R. Interventions for insomnia during pregnancy. Cochrane Database of Systematic Reviews 2014, Issue 10. [DOI: 10.1002/14651858.CD011355]

\section{Morin 2005}

Morin CM, Koetter U, Bastien C, Ware JC, Wooten V. Valerian-hops combination and diphenhydramine for treating insomnia: a randomized placebo-controlled clinical trial. Sleep 2005;28(11):1465-71.

\section{Morin 2006}

Morin CM, LeBlanc M, Daley M, Gregoire JP, Mérette C. Epidemiology of insomnia: prevalence, self-help treatments, consultations, and determinants of help-seeking behaviors. Sleep Medicine 2006;7(2):123-30.

\section{Morin 2012}

Morin CM, Benca R. Chronic insomnia. Lancet 2012;379 (9821):1129-41.

\section{Mowry 2013}

Mowry JB, Spyker DA, Cantilena LR Jr, Bailey JE, Ford M. 2012 Annual Report of the American Association of Poison Control Centers' National Poison Data System (NPDS): 30th Annual Report. Clinical Toxicology (Philadelphia, Pa.) 2013;51(10):949-1229.

\section{NICE 2014}

National Institute for Clinical Excellence. Insomnia. London: National Institute for Clinical Excellence, 2014.

\section{Nutt 2010}

Nutt DJ, Stahl SM. Searching for perfect sleep: the continuing evolution of GABAA receptor modulators as hypnotics. Journal of Psychopharmacology (Oxford, England) 2010;24(11):1601-12.

\section{Ohayon 2002}

Ohayon MM. Epidemiology of insomnia: what we know and what we still need to learn. Sleep Medicine Reviews 2002;6(2):97-111.

\section{Ohayon 2009}

Ohayon MM, Reynolds CF 3rd. Epidemiological and clinical relevance of insomnia diagnosis algorithms according to the DSM-IV and the International Classification of Sleep Disorders (ICSD). Sleep Medicine 2009;10(9):952-60.

\section{Ohayon 2012}

Ohayon MM, Riemann D, Morin C, Reynolds CF 3rd. Hierarchy of insomnia criteria based on daytime consequences. Sleep Medicine 2012;13(1):52-7.

\section{Parrott 1978}

Parrott AC, Hindmarch I. Factor analysis of a sleep evaluation questionnaire. Psychological Medicine 1978;8(2): 325-9.

\section{Perlis 2005}

Perlis ML, Jungquist C, Smith MT, Posner D. Cognitive Behavioral Treatment of Insomnia. New York: Springer, 2005.

\section{Peters 2008}

Peters J, Sutton A, Jones D, Abrams K, Rushton L. Contourenhanced meta-analysis funnel plots help distinguish publication bias from other causes of asymmetry. Journal of Clinical Epidemiology 2008;61(10):991-6.

\section{Poyares 2004}

Poyares D, Guilleminault C, Ohayon MM, Tufik S. Chronic benzodiazepine usage and withdrawal in insomnia patients. Journal of Psychiatric Research 2004;38(3):327-34.

RevMan 2014 [Computer program] Nordic Cochrane Centre, The Cochrane Collaboration. Review Manager (RevMan). Version 5.3. Copenhagen: Nordic Cochrane Centre, The Cochrane Collaboration, 2014.

\section{Richelson 1979}

Richelson E. Tricyclic antidepressants and histamine H1 receptors. Mayo Clinic Proceedings 1979;54(10):669-74.

\section{Riemann 2007}

Riemann D. Insomnia and comorbid psychiatric disorders. Sleep Medicine 2007;8(Suppl 4):S15-S20.

\section{Riemann 2009}

Riemann D, Perlis ML. The treatments of chronic insomnia: a review of benzodiazepine receptor agonists and psychological and behavioral therapies. Sleep Medicine Reviews 2009;13(3):205-14. 
Risberg 1975

Risberg AM, Risberg J, Ingvar DH. Effects of promethazine on nocturnal sleep in normal man. Psychopharmacologia 1975;43(3):279-84.

Rosekind 2010

Rosekind MR, Gregory KB. Insomnia risks and costs: health, safety, and quality of life. American Journal of Managed Care 2010;16(8):617-26.

\section{Roth 2011}

Roth T, Coulouvrat C, Hajak G, Lakoma MD, Sampson NA, Shahly V, et al. Prevalence and perceived health associated with insomnia based on DSM-IV-TR; International Statistical Classification of Diseases and Related Health Problems, Tenth Revision; and Research Diagnostic Criteria/International Classification of Sleep Disorders, Second Edition criteria: results from the America Insomnia Survey. Biological Psychiatry 2011;69(6):592-600.

\section{Rudolph 2011}

Rudolph U, Knoflach F. Beyond classical benzodiazepines: novel therapeutic potential of GABAA receptor subtypes. Nature Reviews. Drug Discovery 2011;10(9):685-97.

\section{Rösner 2013a}

Rösner S, Soyka M, Hajak G, Wehrle R, Englbrecht C. Zaleplon for insomnia. Cochrane Database of Systematic Reviews 2013, Issue 8. [DOI: 10.1002/ 14651858.CD010702]

\section{Rösner 2013b}

Rösner S, Soyka M, Hajak G, Wehrle R, Englbrecht C. Eszopiclone for insomnia. Cochrane Database of Systematic Reviews 2013, Issue 8. [DOI: 10.1002/ 14651858.CD010703]

Rösner 2013c

Rösner S, Soyka M, Hajak G, Wehrle R, Englbrecht C. Zolpidem for insomnia. Cochrane Database of Systematic Reviews 2013, Issue 8. [DOI: 10.1002/ 14651858.CD010700]

Rösner 2013d

Rösner S, Soyka M, Hajak G, Wehrle R, Englbrecht C. Zopiclone for insomnia. Cochrane Database of Systematic Reviews 2013, Issue 8. [DOI: 10.1002/ 14651858.CD010701]

Saddichha 2010

Saddichha S. Diagnosis and treatment of chronic insomnia. Annals of Indian Academy of Neurology 2010;13(2):94-102.

Sakurai 2005

Sakurai T. Roles of orexin/hypocretin in regulation of sleep/ wakefulness and energy homeostasis. Sleep Medicine Reviews 2005;9(4):231-41.

Salanti 2009

Salanti G, Marinho V, Higgins J. A case study of multipletreatments meta-analysis demonstrates that covariates should be considered. Journal of Clinical Epidemiology 2009;62:857-64.

Salanti 2011

Salanti G, Ades A, Ioannidis J. Graphical methods and numerical summaries for presenting results from multiple- treatment meta-analysis: an overview and tutorial. Journal of Clinical Epidemiology 2011;64:163-71.

Salanti 2014

Salanti G, Del Giovane C, Chaimani A, Caldwell DM,

Higgins JP. Evaluating the quality of evidence from a network meta-analysis. PloS One 2014;9(7):e99682.

\section{Schwartz 1999}

Schwartz S, McDowell Anderson W, Cole SR, CornoniHuntley J, Hays JC, Blazer D. Insomnia and heart disease: a review of epidemiologic studies. Journal of Psychosomatic Research 1999;47(4):313-33.

\section{Schünemann 2011}

Schünemann H, Oxman A, Higgins J, Vist G, Glasziou P, Guyatt G. Chapter 11: Presenting results and 'Summary of findings' tables. In: Higgins JPT, Green S editor(s). Cochrane Handbook for Systematic Reviews of Interventions. 5.1.0 [updated March 2011]. The Cochrane Collaboration, 2011

\section{Shekleton 2014}

Shekleton JA, Flynn-Evans EE, Miller B, Epstein LJ, Kirsch D, Brogna LA, et al. Neurobehavioral performance impairment in insomnia: relationships with self-reported sleep and daytime functioning. Sleep 2014;37(1):107-16.

Shibley 2008

Shibley HL, Malcolm RJ, Veatch LM. Adolescents with insomnia and substance abuse: consequences and comorbidities. Journal of Psychiatric Practice 2008;14(3): $146-53$.

Simpson 2008

Simpson D, Curran MP. Ramelteon: a review of its use in insomnia. Drugs 2008;68(13):1901-19.

Stepanski 2003

Stepanski EJ, Wyatt JK. Use of sleep hygiene in the treatment of insomnia. Sleep Medicine Reviews 2003;7(3): 215-25.

Takeshima 2014

Takeshima N, Furukawa TA, Hayasaka Y, Ogawa Y, Tajika A. Ramelteon for insomnia. Cochrane Database of Systematic Reviews 2014, Issue 3. [DOI: 10.1002/ 14651858.CD011049]

Turner 2012

Turner RM, Davey J, Clarke MJ, Thompson SG, Higgins JPT. Predicting the extent of heterogeneity in meta-analysis, using empirical data from the Cochrane Database of Systematic Reviews. International Journal of Epidemiology 2012;41(3):818-27.

Veroniki 2013

Veroniki AA, Vasiliadis HS, Higgins JP, Salanti G. Evaluation of inconsistency in networks of interventions. International Journal of Epidemiology 2013;42(1):332-45.

\section{Vgontzas 2009a}

Vgontzas AN, Liao D, Bixler EO, Chrousos GP, Vela-Bueno A. Insomnia with objective short sleep duration is associated with a high risk for hypertension. Sleep 2009;32(4):491-7.

Comparative efficacy and acceptability of pharmacological treatments for insomnia in adults: a systematic review and network meta- 
Vgontzas 2009b

Vgontzas AN, Liao D, Pejovic S, Calhoun S, Karataraki M, Bixler EO. Insomnia with objective short sleep duration is associated with type 2 diabetes: a population-based study. Diabetes Care 2009;32(11):1980-5.

Wafford 2008

Wafford KA, Ebert B. Emerging anti-insomnia drugs: tackling sleeplessness and the quality of wake time. Nature Reviews. Drug Discovery 2008;7(6):530-40.

Ware 1983

Ware JC. Tricyclic antidepressants in the treatment of insomnia. Journal of Clinical Psychiatry 1983;44(9 Pt 2): $25-8$.

Ware 1992

Ware JE Jr, Sherbourne CD. The MOS 36-item short-form health survey (SF-36). I. Conceptual framework and item selection. Medical Care 1992;30(6):473-83.

\section{Weber 2010}

Weber J, Siddiqui MA, Wagstaff AJ, McCormack PL. Lowdose doxepin: in the treatment of insomnia. CNS Drugs 2010;24(8):713-20.

White 2011

White IR. Multivariate random-effects meta-regression: updates to mvmeta. Stata Journal 2011;11:255-70.

White 2012

White IR, Barrett JK, Jackson D, Higgins JPT. Consistency and inconsistency in network meta-analysis: model estimation using multivariate meta-regression. Research

Synthesis Methods 2012;3(2):111-25.

\section{WHO 1992}

World Health Organization. The Tenth Revision of the International Classification of Diseases and Related Health Problems (ICD-10). Geneva: World Health Organization, 1992.

\section{Wickwire 2015}

Wickwire EM, Shaya FT, Scharf SM. Health economics of insomnia treatments: the return on investment for a good night's sleep. Sleep Medicine Reviews 2015;28(30):72-82.

Winkler 2014

Winkler A, Auer C, Doering BK, Rief W. Drug treatment of primary insomnia: a meta-analysis of polysomnographic randomized controlled trials. CNS Drugs 2014;28(9): 799-816.

\section{Yeung 2015}

Yeung WF, Chung KF, Yung KP, Ng TH. Doxepin for insomnia: a systematic review of randomized placebocontrolled trials. Sleep Medicine Reviews 2015;19:75-83.

\section{Zisapel 2003}

Zisapel N, Laudon M. Subjective assessment of the effects of CNS-active drugs on sleep by the Leeds sleep evaluation questionnaire: a review. Human Psychopharmacology 2003; 18(1):1-20. [PUBMED: 12532311]

* Indicates the major publication for the study

\section{A P P E N D I C E S}

\section{Appendix I. Search strategies}

\section{Cochrane Library}

Databases on the Cochrane Library (CDSR, CENTRAL, DARE, HTA and NHS-EED) will be searched using the following strategy (note specific list of drug terms):

\#1 insomni*:ti

\#2 MeSH descriptor: [Sleep Initiation and Maintenance Disorders] explode all trees and with qualifier(s): [Drug therapy - DT]

\#3 insomni*

\#4 sleep near/2 disorder*

\#5 MeSH descriptor: [Sleep Disorders] explode all trees and with qualifier(s): [Drug therapy - DT]

\#6 MeSH descriptor: [Sleep Initiation and Maintenance Disorders] explode all trees

\#7 (\#3 or \#4 or \#5 or \#6)

\#8 (amitriptyline or doxepin or mirtazapine or trazodone)


or lorazepam or lormetazepam or midazolam or nimetazepam or nitrazepam or oxazepam or quazepam or rilmazafone or temazepam or triazolam)

\#10 (nonbenzodiazepin* or non-benzodiazepin* or "Z drug*” or eszopiclon* or zaleplon or zolpidem or zopiclon*)

Comparative efficacy and acceptability of pharmacological treatments for insomnia in adults: a systematic review and network meta- 
\#11 (melatonin* or ramelteon)

\#12 (orexin or suvorexant)

$\# 13$ (\#8 or \#9 or \#10 or \#11 or \#12)

$\# 14$ (\#7 and \#13)

\#15 (pharmacotherap* or antidepress* or anti depress* or benzodiazepin* or melaton*):ti

\#16 MeSH descriptor: [Antidepressive Agents] explode all trees and with qualifier(s): [Administration \& dosage - AD, Therapeutic use - TU]

\#17 MeSH descriptor: [Benzodiazepines] explode all trees and with qualifier(s): [Administration \& dosage - AD, Therapeutic use TU]

\#18 MeSH descriptor: [Hypnotics and Sedatives] explode all trees and with qualifier(s): [Administration \& dosage - AD, Therapeutic use - TU]

$\# 19$ (\#15 or \#16 or \#17 or \#18)

$\# 20$ (\#1 or \#6) and \#19

$\# 21$ (\#2 or \#14 or \#20)

\section{OVID databases}

Search strategies for MEDLINE, Embase and PsycINFO have been designed to reduce the identification of a large number of irrelevant hits, e.g. treatment studies for depression or other common mental disorders where the symptoms of insomnia are discussed in terms of association, risk or as an adverse effect of psychotropic medication. The searches have also been tailored to exploit the nature and scope of each biomedical database.

We will search OVID MEDLINE (1946 onwards) using the following combination of terms:

1. insomni*.ti,kf.

2. "Sleep Initiation and Maintenance Disorders"/dt [Drug Therapy]

3. Sleep Wake Disorders/dt [Drug Therapy, Therapy]

4. Sleep/dt,th [Drug Therapy]

5. insomni*.ti,ab,kf.

6. (sleep adj2 disorder*).ti,ab,kf.

7. exp "Sleep Initiation and Maintenance Disorders"/

8. (Pittsburgh Sleep Quality Index or PSQI or Insomnia Severity Index or Leeds Sleep Evaluation Questionnaire or LSEQ or (insomnia adj2 (evaluation or index or measure* or questionnaire or rating or scale or schedule or subscale))).ab.

9. or $/ 5-8$

10. (amitriptyline or doxepin or mirtazapine or trazodone).mp.

11. (benzodiaz* or brotizolam or clonazepam or diazepam or estazolam or flunitrazepam or flurazepam or haloxazolam or loprazolam or lorazepam or lormetazepam or midazolam or nimetazepam or nitrazepam or oxazepam or quazepam or rilmazafone or temazepam or triazolam).ti,ab,kf,rn, nm.

12. (nonbenzodiazepin* or non-benzodiazepin* or "Z drug*” or eszopiclon* or zaleplon or zolpidem or zopiclon*).mp.

13. (melatonin* or ramelteon).mp.

14. (orexin or suvorexant).mp.

15. or/10-14

16. 9 and 15

17. benzodiazepines/ad,tu or clonazepam/ad,tu or diazepam/ad,tu or flunitrazepam/ad,tu or flurazepam/ad,tu or lorazepam/ad,tu or nitrazepam/ad,tu or temazepam/ad,tu or estazolam/ad,tu or midazolam/ad,tu or triazolam/ad,tu

18. exp Antidepressive Agents/ad,tu,th [Administration \& Dosage, Therapeutic Use, Therapy]

19. "Hypnotics and Sedatives"/ad,tu,th [Administration \& Dosage, Therapeutic Use, Therapy]

20. (pharmacotherap* ${ }^{*}$ or antidepress* or anti depress* or benzodiazepin* or melaton*).ti,kf.

21. or/ $17-20$

22. ( 1 or 7$)$ and 21

23. controlled clinical trial.pt.

24. randomized controlled trial.pt.

25. (randomi\#ed or randomi\#ation).ab,ti.

26. randomly.ab.

Comparative efficacy and acceptability of pharmacological treatments for insomnia in adults: a systematic review and network meta- 
27. (random* adj3 (administ* or allocat* or assign* or class* or control* or determine* or divide* or distribut* or expose* or fashion or

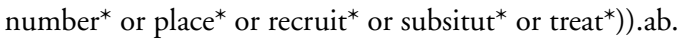

28. placebo*.ab,ti.

29. trial.ab,ti.

30. groups.ab.

31. ((singl* ${ }^{*}$ or doubl* or tripl* or trebl*) adj3 (blind* or mask* or dummy*)).mp.

32. exp animals/ not (humans.sh. and exp animals/)

33. or $/ 23-31$

34. 33 not 32

35. ( 2 or 3 or 4 or 16 or 22$)$ and 34

OVID Embase will be searched (1980 onwards) using the following combination of terms:

1. *insomnia/

2. insomnia/dt

3. sleep disorder/dt

4. insomni*.ti,kw.

5. exp insomnia/

6. Insomnia Severity Index/

7. insomni*.ti,ab,kw.

8. (Pittsburgh Sleep Quality Index or PSQI or Insomnia Severity Index or Leeds Sleep Evaluation Questionnaire or LSEQ or (insomnia adj2 (evaluation or index or measure* or questionnaire or rating or scale or schedule or subscale))).ab.

9. or/5-8

10. (amitriptyline or doxepin or mirtazapine or trazodone).mp.

11. (benzodiaz* or brotizolam or clonazepam or diazepam or estazolam or flunitrazepam or flurazepam or haloxazolam or loprazolam or lorazepam or lormetazepam or midazolam or nimetazepam or nitrazepam or oxazepam or quazepam or rilmazafone or temazepam or triazolam).ti,ab,kw,rn.

12. brotizolam/ or clonazepam/dt or diazepam/dt or estazolam/ or flunitrazepam/dt or flurazepam/dt or haloxazolam/ or loprazolam/ or lorazepam/dt or lormetazepam/ or midazolam/dt or nimetazepam/ or quazepam/ or rilmazafone/ or temazepam/ or triazolam/dt

13. * clonazepam/ or *diazepam/ or *flunitrazepam/ or *flurazepam/ or *lorazepam/ or *midazolam/ or *temazepam/ or *triazolam/

14. (nonbenzodiazepin* or non-benzodiazepin* or "Z drug*” or eszopiclon* or zaleplon or zolpidem or zopiclon*).mp.

15. eszopiclone/dt or zopiclone/dt or zolpidem/dt or zolpidem tartrate/dt or zaleplon/dt

16. *eszopiclone/ or *zopiclone/ or *zolpidem/ or *zolpidem tartrate/ or *zaleplon/

17. (melatonin* or ramelteon).mp.

18. (orexin or suvorexant).mp.

19. or/10-18

20. randomized controlled trial.de.

21. randomization.de.

22. placebo.de.

23. placebo\$.ti,ab.

24. randomi\#ed.ti,ab.

25. randomly.ab.

26. ((singl\$ or doubl\$ or trebl\$ or tripl\$) adj3 (blind $\$$ or mask\$ or dummy)).mp.

27. ((animal or nonhuman) not (human and (animal or nonhuman))).de.

28. or/20-26

29. 28 not 27

30. 9 and 19 and 29

31. limit 30 to exclude medline journals

32. ( 1 or 2 or 3 or 4$)$ and 19 and 29

33. 33 or 34

OVID PsycINFO will be searched (all years) using the following combination of terms:

1. insomnia/

2. insomni*.ti,ab,id.

Comparative efficacy and acceptability of pharmacological treatments for insomnia in adults: a systematic review and network meta- 
3. (Pittsburgh Sleep Quality Index or PSQI or Insomnia Severity Index or ISI or Leeds Sleep Evaluation Questionnaire or LSEQ or (insomnia adj2 (evaluation or index or measure* or questionnaire or rating or scale or schedule or subscale))).tm.

4. or/1-3

5. sleep disorders/

6. (insomni* or (sleep adj2 disorder*)).ti,id.

7. “3340”.cc. [Classification Code=Clinical Psychopharmacology]

8. ((1 or 5 or 6$)$ and 7$)$

9. (amitriptyline or doxepin or mirtazapine or trazodone).ti,ab,id,sh.

10. (benzodiaz* or brotizolam or clonazepam or diazepam or estazolam or flunitrazepam or flurazepam or haloxazolam or loprazolam or lorazepam or lormetazepam or midazolam or nimetazepam or nitrazepam or oxazepam or quazepam or rilmazafone or temazepam or triazolam).ti,ab,id,sh.

11. (nonbenzodiazepin* or non-benzodiazepin* or "Z drug*” or eszopiclon* or zaleplon or zolpidem or zopiclon*).ti,ab,id,sh.

12. (melatonin* or ramelteon).ti,ab,id,sh.

13. (orexin or suvorexant).ti,ab,id,sh.

14. or/9-13

15. (pharmacotherap* or antidepress* or anti depress* or benzodiazepin* or melaton*).ti.

16. exp antidepressant drugs/

17. exp benzodiazepines/

18. exp hypnotic drugs/

19. drug therapy/

20. or/15-19

21. treatment effectiveness evaluation.sh.

22. clinical trials.sh.

23. placebo.sh.

24. placebo.ti,ab,id.

25. randomly.ab.

26. randomi\#ed.ti,ab,id.

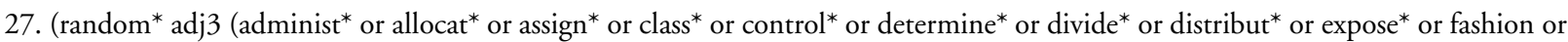

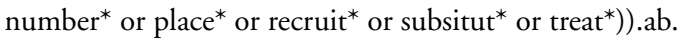

28. trial.ti,ab,id.

29. ((singl* ${ }^{*}$ or doubl* or trebl* or tripl*) adj3 (blind* or mask* or dummy)).ti,ab,id.

30. (control* adj3 group*).ab.

31. "2000”.md. [Methodology=Treatment Outcome/Clinical Trial]

32. or/21-31

33. (4 and 14 and 32)

34. (8 and 32)

35. (6 and 20 and 32 )

36. or/33-35

PubMed will be searched (current year only) using the following terms:

((insomni* OR sleep disorder* OR sleep wake disorder* OR sleep initiation OR sleep onset OR sleep latency) AND (amitriptyline OR doxepin OR mirtazapine OR trazodone OR benzodiazepin* OR brotizolam OR clonazepam OR diazepam OR estazolam OR flunitrazepam OR flurazepam OR haloxazolam OR loprazolam OR lorazepam OR lormetazepam OR midazolam OR nimetazepam OR nitrazepam OR oxazepam OR quazepam OR rilmazafone OR temazepam OR triazolam OR nonbenzodiazepin* OR nonbenzodiazepin* OR "Z drug” OR "Z drugs" OR eszopiclon* OR zaleplon OR zolpidem OR zopiclon* OR melatonin* or ramelteon OR orexin OR suvorexant) AND (random* OR placebo OR trial OR (control* AND group) OR treat*[Title] OR efficacy[Title] OR effectiveness[Title)) Filters: Publication date from 2015/01/01 to 2016/01/31

\section{International Trial Registries:}

The World Health Organisations trials portal (ICTRP) will be searched using the following search string:

(insomnia and amitriptyline or insomnia and doxepin or insomnia and mirtazapine or insomnia and trazodone or insomnia and brotizolam or insomnia and clonazepam or insomnia and diazepam or insomnia and estazolam or insomnia and flunitrazepam or insomnia and flurazepam or insomnia and haloxazolam or insomnia and loprazolam or insomnia and lorazepam or insomnia and lormetazepam or insomnia and midazolam or insomnia and nimetazepam or insomnia and nitrazepam or insomnia and quazepam or insomnia and rilmazafone or insomnia

Comparative efficacy and acceptability of pharmacological treatments for insomnia in adults: a systematic review and network meta17 
and temazepam or insomnia and triazolam or insomnia and eszopiclone or insomnia and zaleplon or insomnia and zolpidem or insomnia and zopiclone or insomnia and melatonin or insomnia and ramelteon or insomnia and orexin or insomnia and suvorexant)

An advanced search of ClinicalTrials.gov will be conducted, for:

CONDITION=insomnia and STUDY TYPE=interventional

Results will be imported into Excel and filtered by INTERVENTION=drug and STUDY DESIGNS=randomized

\section{WHAT'S NEW}

\begin{tabular}{l|l|l}
\hline Date & Event & Description \\
\hline 26 September 2016 & Amended & Contact details updated. \\
\hline
\end{tabular}

\section{CONTRIBUTIONSOFAUTHORS}

All of the authors actively contributed to the development of this protocol.

\section{DECLARATIONSOF INTEREST}

Franco De Crescenzo, Francesca Foti, Marco Ciabattini, Cinzia Del Giovane, Monica Sañé Schepisi, Digby Quested, Andrea Cipriani, Corrado Barbui, Laura Amato have no conflict of interest to declare relating to this work.

Norio Watanabe has research funds from the Japanese Ministry of Health Labor and Welfare and the Japanese Ministry of Education, Science, and Technology. He has also received royalties from Sogensha and Paquet, and speaking fees and research funds from Asahi Kasei, Dai-Nippon Sumitomo, Eli Lilly, GlaxoSmithKline, Janssen, Meiji, MSD, Otsuka and Pfizer during the last five years. 